This item was submitted to Loughborough's Research Repository by the author.

Items in Figshare are protected by copyright, with all rights reserved, unless otherwise indicated.

\title{
Large eddy simulation and PIV measurements of unsteady premixed flames accelerated by obstacles
}

PLEASE CITE THE PUBLISHED VERSION

http://dx.doi.org/10.1007/s10494-008-9198-3

\section{PUBLISHER}

(C) Springer Science+Business Media B.V.

\section{VERSION}

AM (Accepted Manuscript)

\section{LICENCE}

CC BY-NC-ND 4.0

\section{REPOSITORY RECORD}

Di Sarli, Valeria, Almerinda Di Benedetto, G. Russo, Simon Jarvis, Edward J. Long, and Graham K. Hargrave. 2019. "Large Eddy Simulation and PIV Measurements of Unsteady Premixed Flames Accelerated by Obstacles". figshare. https://hdl.handle.net/2134/13447. 
This item was submitted to Loughborough's Institutional Repository (https://dspace.lboro.ac.uk/) by the author and is made available under the following Creative Commons Licence conditions.

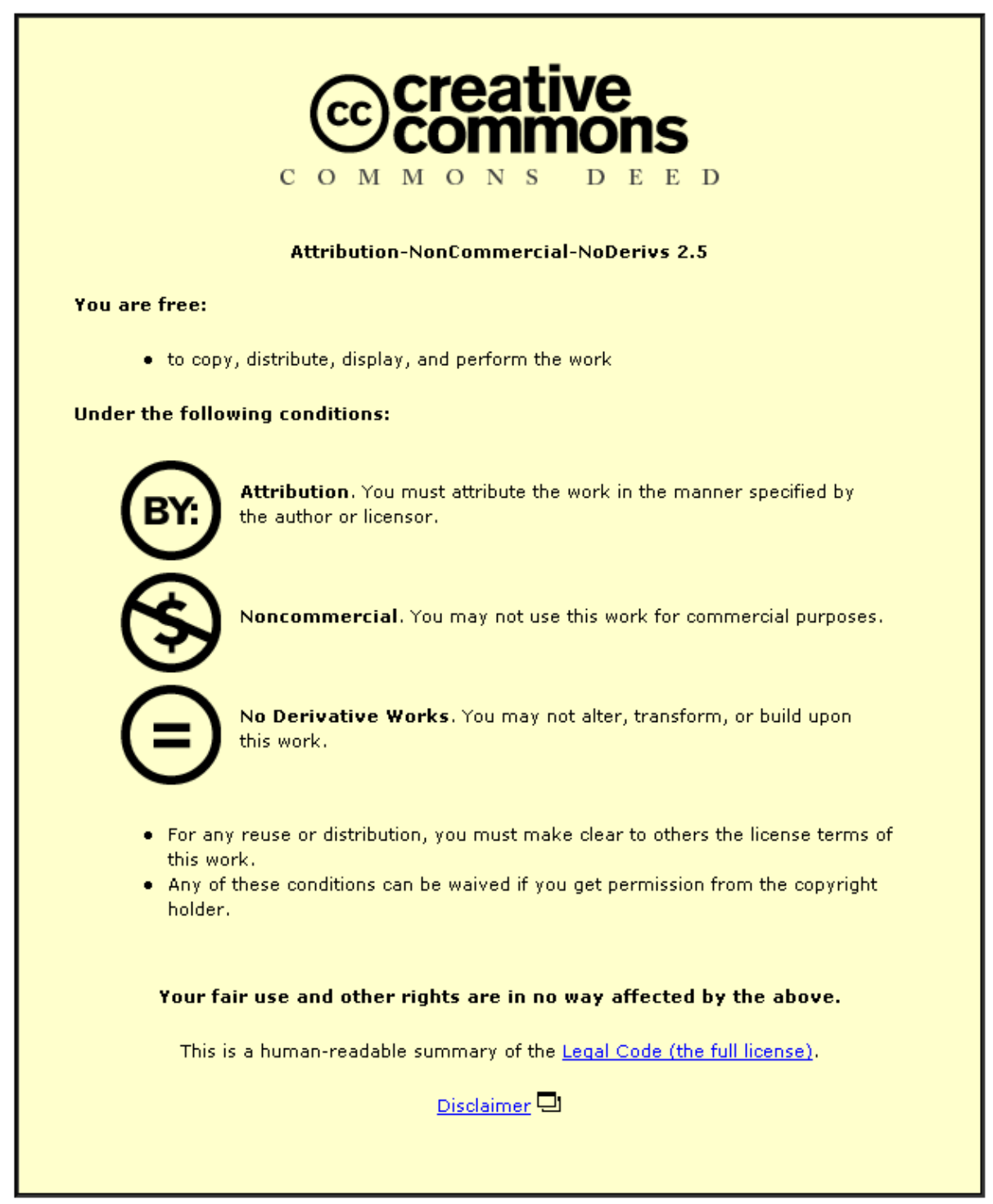

For the full text of this licence, please go to: http://creativecommons.org/licenses/by-nc-nd/2.5/ 


\title{
Large Eddy Simulation and PIV Measurements of Unsteady Premixed Flames Accelerated by Obstacles
}

\author{
V. Di Sarli ${ }^{1}$, A. Di Benedetto ${ }^{1}$, G. Russo ${ }^{2}$, S. Jarvis ${ }^{3}$, E.J. Long ${ }^{3}$, G.K. Hargrave ${ }^{3}$ \\ ${ }^{1}$ Istituto di Ricerche sulla Combustione - CNR, Via Diocleziano 328, 80124, Napoli, Italy \\ ${ }^{2}$ Dipartimento di Ingegneria Chimica - Università degli Studi di Napoli Federico II, Piazzale Tecchio 80, \\ 80125, Napoli, Italy \\ ${ }^{3}$ Wolfson School of Mechanical and Manufacturing Engineering, Loughborough University, \\ Loughborough, Leicestershire, LE11 3TU, United Kingdom
}

Short Title: LES Modelling and PIV Measurements of Unsteady Premixed Combustion

\section{Corresponding author:}

Dr. Valeria Di Sarli

Phone: +39 0817622673

Fax: +39 0817622915

Email: disarli@irc.cnr.it 


\title{
Large Eddy Simulation and PIV Measurements of Unsteady Premixed Flames Accelerated by Obstacles
}

\author{
V. Di Sarli ${ }^{1}$, A. Di Benedetto ${ }^{1}$, G. Russo ${ }^{2}$, S. Jarvis ${ }^{3}$, E.J. Long ${ }^{3}$, G.K. Hargrave ${ }^{3}$ \\ ${ }^{1}$ Istituto di Ricerche sulla Combustione - CNR, Via Diocleziano 328, 80124, Napoli, Italy \\ ${ }^{2}$ Dipartimento di Ingegneria Chimica - Università degli Studi di Napoli Federico II, Piazzale Tecchio 80, \\ 80125, Napoli, Italy
}

3 Wolfson School of Mechanical and Manufacturing Engineering, Loughborough University, Loughborough, Leicestershire, LE11 3TU, United Kingdom

\begin{abstract}
In gas explosions, the unsteady coupling of the propagating flame and the flow field induced by the presence of blockages along the flame path produces vortices of different scales ahead of the flame front. The resulting flame/vortex interaction intensifies the rate of flame propagation and the pressure rise.

In this paper, a joint numerical and experimental study of unsteady premixed flame propagation around three sequential obstacles in a small scale vented explosion chamber is presented. The modelling work is carried out utilising Large Eddy Simulation (LES). In the experimental work, previous results [Patel, S.N.D.H., Jarvis, S., Ibrahim, S.S., Hargrave, G.K., Proceedings of the Combustion Institute 29, 1849-1854 (2002)] are extended to include simultaneous flame and Particle Image Velocimetry (PIV) measurements of the flow field within the wake of each obstacle.

Comparisons between LES predictions and experimental data show a satisfactory agreement in terms of shape of the propagating flame, flame arrival times, spatial profile of the flame speed, pressure time history and velocity vector fields.

Computations through the validated model are also performed to evaluate the effects of both large scale and sub-grid scale (sgs) vortices on the flame propagation. The results obtained demonstrate that the large vortical structures dictate the evolution of the flame in qualitative terms (shape and structure of the flame, succession of the combustion regimes along the path, acceleration-deceleration step around each obstacle, pressure time trend). Conversely, the sgs vortices do not affect the qualitative trends. However, it is essential to model their effects on the combustion rate to achieve quantitative predictions for the flame speed and the pressure peak.
\end{abstract}

Keywords: Large Eddy Simulation, Particle Image Velocimetry, Unsteady Propagation, Premixed Combustion, Obstacles, Sub-Grid Scale Turbulence. 


\section{Introduction}

In practical gas explosions, flames propagating away from an ignition source often encounter obstacles (vessels, pipes, tanks, flow cross-section variations, instrumentations, etc...) along their path. The unsteady coupling of the moving flame and the flow field induced by the presence of local blockage produces vortices of different scales ahead of the flame front. These vortices disturb the flat propagation of the flame, increasing its rate of propagation and the pressure rise.

During its progression, the flame experiences various combustion regimes [1-11]. Initially, a weak turbulence, which is not able to affect the flame propagation, develops. From this, the increasing turbulence level generated by the propagation itself allows the vortices formed ahead of the front to wrinkle the flame, increasing the flame surface area. Eventually, the vortices may also enter the flame structure, enhancing the transport of heat and mass in the preheating zone or disrupting/quenching the flame.

The transient flame/vortex interaction is the key process in the description of an explosive phenomenon [2,5,7-10]. Consequently, the study of the unsteady premixed flame propagation through obstacles has to focus on measurements/simulations of the dynamic evolution of the flame, vortices and their coupling.

Over the last decade, progress within the field of optical diagnostics has produced tools that can provide data containing high levels of both spatial and temporal resolution [1215]. These tools have allowed sequences of flame images, flame speed profiles, maps of velocity vectors, turbulence characteristics and species concentrations to be measured without disturbing the interactions being investigated. 
High-Speed Laser Sheet Flow Visualisation (HSLSFV) and Particle Image Velocimetry (PIV) are two of the most applicable techniques for characterising the flame/flow interaction. Through HSLSFV images, the progression of the propagating flame is captured, thus obtaining qualitative information about flame shape and scales of flame front wrinkling [1,3-7,9-11]. PIV allows the measurement of the velocity field ahead of the flame front, leading to the quantification of the flame/vortex interaction $[10,16,17]$.

On the numerical side, thanks to the growing computational power and the availability of distributed computing algorithms, Large Eddy Simulation (LES) is emerging as a useful method for the prediction of turbulent reacting flows [18-20]. The attraction of LES is that it offers an improved representation of turbulence, and the resulting flame/turbulence interaction, with respect to classical RANS approaches.

Both PIV and LES have been proven to be successful techniques in steady-state problems such as those encountered in combustors and burners [21-26]. Additionally, they also appear to be promising tools for studying explosions [5,7-10].

PIV provides the measurement of large scale vortices, LES their numerical simulation. LES directly resolves all of the large turbulent structures up to the grid dimension, while models the small sub-grid structures that, however, exhibit a more "universal" behaviour. Unfortunately, chemical reactions in combustion processes occur at the smallest unresolved turbulent scales. Hence, the effect of the small vortices on the combustion rate has to be taken into account by means of sub-grid scale (sgs) combustion models.

Most of the LES combustion models have been developed and tested for applications in which a stationary turbulent combustion regime is established [18-20]. Recently, Richard et al. [27] have proposed the solution of a transport equation for the sub-grid 
scale flame surface density (i.e., the sgs flame surface per unit volume) to handle nonequilibrium situations in LES of unsteady combustion during spark ignition engine cycles.

Large eddy simulations of explosions in the presence of obstacles have been performed by Masri and co-workers [5,8] adopting the algebraic closure for the sgs flame surface density by Boger et al. [28]. Although this model exhibits a weak dependence of the combustion rate on the unresolved vortices, the results obtained show very good predictions for the shape and structure of the flame as it propagates through the obstructions. However, the authors have recognised the need for a more sophisticated sgs combustion model to achieve further improvements in quantitative terms (flame speeds and pressure peaks).

For LES of unsteady flames accelerated by obstacles, it is then unclear whether the large vortical structures are dominant or what impact the small vortices have at the subgrid level.

The present paper fits in this framework with the aim at gaining insight into the process of flame/vortex interaction in the unsteady premixed flame propagation around obstacles. This is performed through the conjoined application of LES modelling and PIV measurements.

This paper describes the work undertaken to:

1) Develop an LES model and thoroughly validate it by comparing the numerical results to detailed experimental data;

2) Quantify the roles played by the large scale vortices and the sgs vortices in affecting the flame propagation as simulated by LES. 
To this end, our previous experimental results (HSLSFV images, spatial profile of the flame speed, pressure time history), obtained in a small scale vented explosion chamber containing three repeated obstacles [1], have been here extended to include simultaneous flame and PIV measurements of the flow field within the wake of each obstacle.

In the following, the details of the experimental set-up and the LES model are first described. Then, the results are presented and discussed, starting from the comparison between experimental data and model predictions.

Once validated, the LES model is used to understand the role of the resolved large scale vortices in relation to that of the sgs vortices. More precisely, LES computations are also run with the effect of the sgs combustion model eliminated. The role of the large vortices is then studied separately from that of the small vortices, thus quantifying the relevance of the sgs combustion modelling.

\section{Experimental Work}

The explosion chamber, shown in Fig. 1, and the High-Speed Laser Sheet Flow Visualisation (HSLSFV) technique employed in this study have been described previously [1].

Briefly, the combustion chamber was a $150 \mathrm{~mm}$ x $150 \mathrm{~mm}$ x $500 \mathrm{~mm}$ volume constructed from polycarbonate to provide optical access. Three obstacles (150 mm x $75 \mathrm{~mm}$ x $12 \mathrm{~mm}$ ) were positioned at $100 \mathrm{~mm}$ spacings within the chamber. The bottom end of the chamber was fully closed. The upper end was sealed by a thin PVC membrane that ruptured during the combustion process, allowing the burned gases to escape. 
A stoichiometric mixture of methane and air was purged through the chamber until the entire volume was uniformly filled. The solenoid valves at the inlet and exit of the chamber were then closed and the mixture allowed to settle until the flow structures induced during the filling process had dissipated. After this, the reactant mixture was ignited at the centre of the closed end. Ignition was provided by a simple automotive type spark plug and coil arrangement that provided an ignition energy in the order of 35 - 40 mJ with a dwell time of 4 ms.

The HSLSFV technique applied to image the flame propagation consisted of an Oxford Lasers copper vapour laser synchronised to a Kodak Ektapro 4540 high-speed motion analyser. The output of the copper vapour laser was formed into a $1 \mathrm{~mm}$ thick sheet and introduced into the combustion chamber to illuminate the centre of the rig. The methane-air mixture entering the chamber was seeded with 1 - 2 micron sized droplets of olive oil. This seed material then scattered the light from the laser sheet, enabling an image to be picked up by the high-speed camera located perpendicular to the light sheet. As the flame propagated through the combustion chamber, the oil droplets were consumed, differentiating the regions of burned and unburned gas from each other and highlighting the flame front. The image recording was initiated on ignition of the charge, with a recording rate of 9000 frames per second at a resolution of 256 by 128 pixels.

From the high-speed video sequence, the flame speed as a function of the axial distance from the ignition face was also derived. Particularly, the speed was calculated at the tip of the flame front where the maximum distance from the bottom end was reached. 
The pressure time history was taken from recordings obtained using a high-speed piezoelectric pressure transducer located close to the point of ignition. The pressure was recorded at a rate of $4500 \mathrm{~Hz}$.

To quantify the flame/vortex interaction, Digital Particle Image Velocimetry (DPIV) was employed (Fig. 1). Illumination was provided by a twin-cavity Nd:YAG laser which supplied the two light pulses required for each PIV measurement. The laser light was formed into a vertical sheet measuring $100 \mathrm{~mm}$ by $1 \mathrm{~mm}$ in the image region, the plane of which was aligned to the central axis of the combustion chamber. The laser pulse separation was set according to the peak flow velocity in the area examined. In the region around the first obstacle a time separation of $65 \mu \mathrm{m}$ was used, around the second $25 \mu \mathrm{m}$ and around the third $12 \mu \mathrm{m}$.

The image recording was achieved using a twin frame CCD camera (TSI PIV-Cam with a resolution of $1000 \times 1016$ pixels) imaging regions of $85 \mathrm{~mm}$ by $85 \mathrm{~mm}$ in the flow field.

The complete PIV system was mounted on a vertical traverse, enabling the measurement field to be moved relative to the combustion chamber. This meant that the flow around each obstacle could be measured without realigning the PIV equipment.

The particle image pairs captured were analysed using a two-dimensional Fast Fourier Transform (FFT) cross-correlation routine with a Gaussian peak search algorithm. An interrogation region window of 32 x 32 pixels with a $50 \%$ overlap was used.

The flow tracing particles used for the DPIV technique were the same 1 - 2 micron sized particles of olive oil as used for the laser-sheet flow visualisation, but their number density was significantly reduced so that only around 10-15 particle images appeared in each interrogation region. 
The experiment demonstrated a high level of reproducibility with flame shapes and speeds being directly comparable between different combustion events. However, there was a slight variation in the time taken for the flame to reach the first obstacle of $+/-$ $0.5 \mathrm{~ms}$. This variation, which was maintained throughout the combustion process, was linked directly to the time taken for early flame kernel development.

As a result of this repeatability in combustion behaviour, the pressure traces demonstrated little deviations between events other than the temporal shift of +/- $0.5 \mathrm{~ms}$ with respect to ignition.

Due to the short time durations involved, only one PIV velocity field could be recorded per combustion event. However, due to the repeatable nature of the experiment, multiple events could be conducted with the velocity data recorded at different positions and times relative to ignition. The motion (and interaction) of the large scale vortices present was found to be consistent between separate combustion events. The variations of the small scale turbulence led to slight variations in the PIV fields.

\section{Mathematical Model Development}

Unsteady compressible flows with premixed combustion are governed by the reactive Navier-Stokes equations, i.e., the conservation equations for mass, momentum, energy and species, joined to the constitutive and state equations.

Under the assumptions of a "flamelet" regime of combustion [29], one-step global irreversible reaction and a unit Lewis number, the species transport equation may be recast in the form of a transport equation for the reaction progress variable $(c)(c=0$ within fresh reactants and $c=1$ within burned products) [30]: 


$$
\frac{\partial \rho c}{\partial t}+\nabla(\rho u c)=\nabla(\rho D \nabla c)+\dot{\omega}_{c}
$$

In Eq. (1), the two left hand-side terms correspond to unsteady effects and convective fluxes, while the two right hand-side terms correspond to molecular diffusion and reaction rate, respectively.

The Large Eddy Simulation (LES) technique is based on the concept of spatial filtering to be applied to the governing equations $[19,31]$. The filtering process filters out the eddies whose scales are smaller than the filter width so that the resulting equations govern the dynamics of the large eddies. However, due to the non-linear nature of the governing equations, the filtering operation gives rise to unknown terms that have to be modelled at the sub-grid level [19].

The unknown terms arising from the momentum equation and the energy equation are the sub-grid scale (sgs) stress tensor and the sgs heat flux, respectively.

The LES Favre-filtered (i.e., mass-weighted filtered) c-equation reads:

$$
\frac{\partial \bar{\rho} \tilde{c}}{\partial t}+\nabla \cdot(\bar{\rho} \tilde{u} \tilde{c})+\nabla \cdot[\bar{\rho}(u c-\tilde{u} \tilde{c})]=\overline{\nabla \cdot(\rho D \nabla c)}+\overline{\dot{\omega}}_{c}
$$

where the overbar $(-)$ denotes a filtered quantity and the tilde $(\sqcup)$ a Favre-filtered quantity. In Eq. (2), there are three unknown terms: the sgs reaction progress variable flux (third term on the left hand side), the sgs molecular diffusion (first term on the right hand side) and the sgs reaction rate (second term on the right hand side). 


\section{Sub-Grid Scale (SGS) Models for Stress Tensor and Scalar Fluxes}

In the present work, the closure of the sgs stress tensor was achieved with the dynamic Smagorinsky-Lilly eddy viscosity model [32]. After formulating a scale-similarity assumption, the model coefficients were dynamically calculated during the LES computations by using the information about the local instantaneous flow conditions provided by the smaller scales of the resolved (known) field. This allowed the eddy viscosity to properly respond to the local flow structures. To separate the smaller scales from the resolved field, a "test filter" was needed with a width larger than the LES grid filter width. The test filter used was a box filter with a volume comprising the cell itself plus the neighbouring cells sharing the faces with the centre cell [33]. With the hexahedral mesh employed, the ratio of the test filter scale to the grid filter scale was around 2.

The sgs fluxes of heat and reaction progress variable were modelled through the gradient hypothesis [19]. The sgs turbulent Schmidt and Prandtl numbers were assumed to be constant and equal to 0.7 [34].

\section{$\underline{\text { Sub-Grid Scale (SGS) Model for Flame/Turbulence Interaction }}$}

In LES, the flame front is not resolved on the numerical grid, the premixed flame thickness being smaller than the mesh size used. Consequently, the flame remains a subgrid phenomenon whose coupling with the unresolved turbulence has to be exclusively modelled.

Among the different approaches proposed to handle the flame/turbulence interaction in LES [19], the flame surface density formalism based on the flamelet concept was here chosen. The main assumption in flamelet models is that of a "thin flame sheet" which means that the flame, or at least the reaction zone, is thinner than the small turbulent 
scales, thus remaining laminar. Furthermore, the high gradients within the thin flame allow a balance to be established between molecular transport and chemistry. This implies that diffusive transport and chemical reactions cannot be modelled independently of each other.

Accordingly, the filtered molecular diffusion and reaction rate (right hand-side terms in Eq. 2) were both included in a sgs flame front displacement term, $\overline{\rho w|\nabla c|}$, expressed as:

$\overline{\rho w|\nabla c|}=\overline{\nabla(\rho D \nabla c)}+\overline{\dot{\omega}}_{c}=\langle\rho w\rangle_{s} \Sigma$

where $\sum$ is the sgs flame surface density (i.e., the sgs flame surface per unit volume) and $\langle\rho w\rangle_{s}$ is the sgs surface-averaged mass-weighted displacement speed.

In Eq. (3), $\langle\rho w\rangle_{s}$ was approximated by $\rho_{0} S_{l}$ [35], where $\rho_{0}$ is the fresh gas density and $S_{l}$ is the laminar burning velocity. $\sum$ was expressed as a function of the sgs flame wrinkling factor, $\Xi_{\Delta}$, (i.e., the sgs flame surface divided by the projection of the flame surface in the propagating direction):

$$
\overline{\rho w|\nabla c|}=\langle\rho w\rangle_{S} \Sigma=\rho_{0} S_{l} \Xi_{\Delta}|\nabla \bar{c}|
$$

To take into account the coupling of flame propagation and unresolved turbulence, in Eq. (4), $\Xi_{\Delta}$ was modelled according to the flame wrinkling model by Charlette et al. [36]: 


$$
\Xi_{\Delta}=\left(1+\frac{\Delta}{\eta_{C}}\right)^{\beta}=\left(1+\min \left[\frac{\Delta}{\delta_{f}}, \Gamma\left(\frac{\Delta}{\delta_{f}}, \frac{u_{\Delta}^{\prime}}{S_{l}}, R e_{\Delta}\right) \frac{u_{\Delta}^{\prime}}{S_{l}}\right]\right)^{\beta}
$$

with $\Xi_{\Delta}$ written in terms of a power-law expression involving an inner cut-off scale, $\eta_{c}$, an outer cut-off scale (i.e., the filter scale), $\Delta$, and the $\beta$ parameter as exponent. The inner cut-off length scale, $\eta_{c}$, defined as the inverse mean curvature of the flame, limits the flame wrinkling at the smallest length scales. $\eta_{c}$ was modelled by introducing an efficiency function, $\Gamma$, which takes into account the net straining effect of the turbulent scales smaller than $\Delta$.

In Eq. (5), the presence of $\min$ is due to the fact that the model could predict inner cutoff scales smaller than the laminar flame thickness, $\delta_{f}$. To avoid this problem (i.e., to keep $\eta_{c} \geq \delta_{f}$ ), the expression was clipped at the laminar flame thickness.

A spectral analysis of the Direct Numerical Simulation (DNS) results of elementary flame/vortex interactions by Colin et al. [37] was performed to construct $\Gamma$ as a function of the filter scale to laminar flame thickness ratio, $\Delta / \delta_{f}$, the sgs turbulent velocity to laminar burning velocity ratio, $u^{\prime}{ }_{\Delta} S_{l}$, and the sgs turbulent Reynolds number, $R e_{\Delta}$. Furthermore, $\Gamma$ was corrected to avoid that the eddies whose characteristic speed falls below $S_{l} / 2$ (very slow eddies) wrinkle the flame.

Charlette et al. [36] implemented the sgs combustion model with $\beta=0.5$ in an LES code in the context of the thickened flame approach, and performed simulations of a premixed flame embedded in a time decaying isotropic turbulence in several different parameter ranges. They found a good agreement in terms of total reaction rate against 
DNS results. In addition, comparisons between the predicted overall turbulent flame speed as a function of the root mean square velocity and the experimental data by Abdel-Gayed and Bradley [38] showed a close agreement over a significant range of parameters, which also overshoots the wrinkled regime of combustion.

In the present paper, the values of the sgs model constants and parameters used were those suggested by Charlette et al. [36]. The sgs turbulent velocity, $u_{\Delta}^{\prime}$, was computed from the sgs turbulent viscosity provided by the dynamic Smagorinsky-Lilly eddy viscosity model [32].

\section{Numerical Solution and Problem Formulation}

The model equations were discretised using a finite volume formulation on a tridimensional non-uniform structured grid composed of 930,000 hexahedral cells, with minimum and maximum resolutions equal to 2 and $3 \mathrm{~mm}$, respectively. Smaller cell size was used close to the walls due to the presence of steeper gradients of the solution field. The grid was built by means of the Gambit pre-processor of the Fluent package (version 6.3.26) [39].

In order to check the grid-independence of the LES computations, simulations were also run with a coarser mesh (cell size varied from 3 - $4 \mathrm{~mm}$ ) and a finer mesh (cell size varied from 1 - 2 mm). No appreciable differences were observed between the solutions obtained through the 2 - $3 \mathrm{~mm}$ grid and the finer grid.

The fraction of the total turbulent kinetic energy residing in the unresolved motions, as evaluated according to the definition of the $M$ parameter given by Pope [40], was around 0.15 . Therefore, at the filter width setting, the percentage of the kinetic energy resolved in the LES calculations was about $85 \%$. 
For the spatial discretisation of the model equations, second order bounded central schemes were chosen, in view of their low numerical diffusion coupled to a weak propensity to give unphysical oscillating solutions. The time integration was performed by using the second order implicit Crank-Nicholson scheme.

Adiabatic and no-slip wall boundary conditions were applied at the solid interfaces (bottom and vertical faces of the chamber, faces of the obstacles). To calculate the shear stress at the wall, a blended linear/logarithmic law-of-the-wall was used [33,41]. The values for $y+$ at the first grid point varied in the $3<y+<10$ range.

Outside the combustion chamber, the computational domain was extended to simulate the presence of a dump vessel. This allowed for a more realistic reproduction of the exit of the expanding gas from the combustion chamber into the atmosphere. A condition of fixed static pressure was assigned at the boundaries of this additional domain whose distance from the exit section of the chamber (750 $\mathrm{mm}$ in each direction) allowed minimising the interference between the reflected pressure waves and the pressure field inside the chamber.

The grid was heavily concentrated in the combustion chamber where the number of grid cells was about $90 \%$ of the total number of cells.

Initial conditions had velocity components, energy and reaction progress variable set to zero everywhere. Ignition was obtained by means of a hemispherical patch of hot combustion products at the centre of the closed end.

Simulations of unsteady flame propagation may be sensitive to the characteristics of the initial flame kernel [42]. In order to assess the dependence of the LES results on the ignition description, computations were carried out changing the ignition patch radius, $R_{\text {ign }}=5 \div 7 \mathrm{~mm}$, and sgs turbulent velocity, $u_{\Delta \_i g n}^{\prime}=0 \div 0.41 \mathrm{~m} / \mathrm{s}$. Figure 2 shows the 
time evolution of the flame location at varying the ignition characteristics. The flame location was obtained by measuring the maximum axial distance of the flame front from the ignition face. The overall flame dynamics is found to be affected only at $R_{\text {ign }}=7 \mathrm{~mm}$.

The results described in Section 4 were obtained by setting $R_{i g n}$ and $u_{\Delta_{-} i g n}$ equal to $5 \mathrm{~mm}$ and $0 \mathrm{~m} / \mathrm{s}$, respectively. The patch radius $(=5 \mathrm{~mm})$ was the minimum value needed to get ignition.

For the stoichiometric methane/air flame simulated, the laminar burning velocity was assumed to be constant with pressure and temperature and equal to $0.41 \mathrm{~m} / \mathrm{s}[43,44]$.

The specific heats of the unburned and burned mixtures were approximated as piecewise fifth-power polynomial functions of temperature. The molecular viscosities were calculated according to Sutherland's law for air viscosity.

Computations were performed by means of the segregated solver of the Fluent code (version 6.3.26) [39] adopting the SIMPLE method to treat the pressure/velocity coupling. The code was parallelised on a 64 bit computing Beowulf cluster consisting of 8 dual-CPU nodes (16 processors) each of them being an AMD Opteron 260 with 2 GB of RAM. The solution for each time step required around 20 iterations to converge with the residual of each equation smaller than 6.0E-4. The time needed to complete the run was about 2 days.

\section{Results and Discussion}

In the following, we first present the comparison between numerical and experimental results in terms of shape of the propagating flame, flame arrival times, spatial profile of 
the flame speed, pressure time history and velocity vector maps ahead of the flame front. From this comparison, a thorough validation of the LES model is obtained.

We then use the validated model to investigate the role of the large scale vortices, in relation to that of the small scale vortices, on the features of the flame propagation. To achieve this, large eddy simulations are also run by assuming that the sub-grid wrinkling factor ( $\Xi_{\Delta}$ in Eq. 4 ) is constant and equal to 1 during the whole propagation. Finally, from both PIV and LES data, the combustion regimes along the flame path are quantified.

\subsection{Comparison between LES and Experiment}

Flame Shapes and Arrival Times - The instantaneous images of the flame structure are presented in Fig. 3 as obtained from both the experimental HSLSFV technique (Fig. 3a) and the LES calculations of the reaction progress variable (Fig. 3b). This figure shows the flame as it propagates inside the chamber at different time instants after ignition/initialisation.

In Fig. 3, the LES results corresponding to the case $\Xi_{\Delta}=1$ are also reported (Fig. 3c). These results will be discussed in the next subsection (4.2).

The images of Fig. 3 are compared at the same distances of the flame front from the bottom end of the combustion chamber. Due to limitations in resolution of the visualisation technique employed, the experimental imaged areas cover a part of the chamber including the three obstacles. The whole chamber is shown in the computed fields.

Figure 3 demonstrates that LES predicts well the features of the flame propagation. As in the experiment, the simulation shows the flame impinging onto the first obstacle, 
with an incomplete consumption of the fuel mixture in the upstream chamber zone. It then separates into two opposite flames, one each side of the obstacle. The flames jet downstream of the obstruction and then curl towards the chamber centreline, thus expanding and reconnecting with each other. This same sequence is then repeated, with ever-greater velocities, as the flames cross the second and third obstacles before venting out of the chamber.

The model is able to reproduce the flame arrival times (the root mean square value of the difference in data between experiment and calculations is equal to $0.5 \mathrm{~ms}$ ). In particular, it catches the arrival time at the first obstacle, meaning that the quasi laminar flame propagation upstream of this obstruction [1,9] is correctly taken into account. Also, the progressive intensification of the flame front wrinkling during the propagation through the obstacles is simulated by LES, together with the formation of flame pockets leaving the main front when the flame burns at the wake of the second and third obstructions.

As it will be demonstrated later, these changes of the structure of the flame front are due to the interaction with the turbulent vortices induced behind the obstacles by the flame propagation itself. Depending on the intensity of the flame/vortex interaction, different flame responses are found.

Flame Speed Profile - In Fig. 4, the experimental flame speed profile along the chamber and the corresponding LES profile are shown (the black rectangles along the $\mathrm{x}$ axis indicate the positions of the three obstacles).

As in the experiment, in the computations, the flame speed was evaluated from the time sequence of the flame images as the displacement of the maximum downstream location of the flame front. 
Figure 4 shows that the model is able to qualitatively and quantitatively reproduce the experimental trend, with both the increased flame acceleration past each obstacle and the drop in acceleration, due to the flame expansion, between the obstacles.

It also results that the presence of multiple obstacles along the flame path strongly accelerates the flame whose speed at the third obstacle becomes around ten times higher than that upstream of the first obstruction.

Pressure Time History - In Fig. 5, the experimental pressure time history at the bottom end of the chamber and the corresponding LES trend are compared. The computed results were obtained through Reynolds-average of the instantaneous predictions. The retained time-scale for Reynolds-averaging was the inverse of the sample frequency of the pressure transducer (i.e., 1/4500 Hz).

Figure 5 shows that the model predicts one dominant pressure peak as observed in the experiment. The pressure peak is under estimated (the maximum overpressure is around $20 \%$ lower than the experiment value), probability due to the fact that the effect of the disposable sealing membrane was not simulated. However, the peak is found at around $37 \mathrm{~ms}$ after ignition and this is in agreement with the experimental result. From both the high-speed (Fig. 3a) and computed (Fig. 3b) images, it turns out that at this time the flame has passed the third obstacle. More precisely, the two opposite flames have almost completed their reconnection in the regions between the obstacles and downstream of the last obstruction, thus exiting the chamber.

These results show that the LES calculations support the high rate of pressure rise occurring during the intense turbulent combustion between the obstacles, as shown from the experimental results. In addition, the pressure decay, starting from the time when the main flame front exits the vent end, is reflected in the model. 
Velocity Vector Maps - Figure 6 shows the experimental images of the propagating flame (left) and the instantaneous velocity vector maps ahead of the front (right), as obtained by both PIV measurements (bottom) and LES calculations (top), when the flame passes sequentially over the three obstacles (first obstacle: Fig. 6a; second obstacle: Fig. 6b; third obstacle: Fig. 6c). In the computed images, the reaction progress variable profiles are superimposed on the velocity fields in the regions corresponding to the flame $(0.3 \leq c \leq 1)$.

These results confirm the good agreement between experimental data and numerical predictions. The model captures the features of the flow field ahead of the propagating front, and then the effects of the coupling between flame and flow field.

From both the experimental and numerical images of Fig. 6, it can be seen that recirculation vortices form within the wake of each obstacle. The size and velocity of these vortices increase with each subsequent obstacle.

The vortex formation is a consequence of the expansion of the flame front upstream of the obstacles. This expansion forces the unburned mixture ahead of the flame front, forming a jet through the opening between each obstacle and the containing walls. These jets give rise to the shedding of eddies from the edges of the obstacles [2,5,7-10]. The trends of the vortex size and velocity through the obstacles are a direct result of the positive feedback mechanism established between the combustion-generated flow field and the flame propagation. That is, the larger velocities arising when the first vortex distorts the flame, thus increasing the flame surface area and burning rate, produce stronger (i.e., larger and faster) recirculation regions behind the following obstacle. Then, as the flame burns into the second (stronger) vortex, faster distortion increases the combustion rate, thus producing yet stronger velocities at the third obstacle. This 
mechanism sustains the continuous flame acceleration past the obstacles (Fig. 4) and the corresponding pressure rise up to the time of the flame venting out of the chamber (Fig. 3 and Fig. 5).

Figure 6a details the interaction between the flame front and the vortex at the wake of the first obstacle. The flame exiting the gap between obstacle and sidewall follows the flow streamlines, thus rolling-up and burning towards the chamber centreline.

Figure $6 \mathrm{~b}$ shows a similar flow configuration within the wake region of the second obstacle: the flame front jets past the obstacle and begins to curl into the vortex formed below the flame tip. However, due to the increased jet of the flame and the larger and faster vortex formed behind the second obstacle, the front propagates a greater distance downstream before beginning to move around the axis of rotation in the flow, in comparison to that of the first obstacle (Fig. 6a).

Within the wake region of the third obstacle (Fig. 6c), the flame can be seen to follow a similar progression to that of the second obstacle. However, the jet formed around this obstacle penetrates even further downstream of the obstruction and the vortex generated has greater size and velocity than that formed at the second obstacle (Fig. 6b). This increase in penetration and vortex size and velocity results in the flame tip interacting with the turbulent flow structure at a greater distance from the obstacle in comparison to the previous obstacle.

Global assessment of Fig. 6 then shows the increase in intensity of the flame/vortex interaction through the obstacles due to the increased speed of both flame and flow field along the flame path. 


\subsection{Role of the Large Scale and Sub-Grid Scale (SGS) Vortices}

In Fig. 7, the maps of the vorticity magnitude are shown as calculated on the iso-surface $c=0.1$ during the flame propagation. The vorticity magnitude significantly increases when the flame reaches the second and third obstacles. Correspondingly, the flame shape changes (Fig. 3b). At the first obstacle the flame is compact, as it propagates pushing the vortex ahead of the front and slowly consuming the mixture in the vortex.

On the contrary, at the second and third obstacles the flame shape is significantly modified by its interaction with the enhanced vorticity field: the flame initially tries to propagate around the vortex, but then rapidly consumes the vortex via the flame pockets.

In principle, the propagating flame has to deal with all of the vortices generated by the interaction with the obstacles, from the largest up to the smallest ones. The large vortices wrinkle the flame, thus increasing its surface. Besides wrinkling the flame surface, the small vortices may also enter the flame structure, enhancing the transport of heat and mass in the preheating zone or eventually disrupting/quenching the flame.

In LES, the large vortices are resolved and their effect on the flame propagation is directly taken into account. The effect of the small vortices (i.e., the sgs vortices) is here quantified through the sub-grid scale wrinkling factor ( $\Xi_{\Delta}$ in Eq. 4) according to the combustion model by Charlette et al. [36] (Eq. 5).

Figure 8 shows the field profiles of the sgs wrinkling factor, $\Xi_{\Delta}$ (Fig. 8a), and the Karlovitz number, $K a=\left[\left(u^{\prime} \Delta S_{l}\right)^{3} \times\left(\delta_{f} / \Delta\right)\right]^{1 / 2}$ (Fig. 8b), both conditioned on the reaction rate, at different propagation stages. 
From Fig. 8a, it can be seen that the values of $\Xi_{\Delta}$ range between 1 and 3. However, the higher values of $\Xi_{\Delta}(\approx 3)$ are reached starting from the second obstacle and only in correspondence of the flame tips, the most of the flame being at lower values. It is then interesting to clarify whether these peak values of $\Xi_{\Delta}$ at the leading edges are relevant for the flame propagation around the obstacles.

Figure $8 \mathrm{~b}$ shows that, although higher values are attained in limited flame zones, the $\mathrm{Ka}$ number ranges between 0 and 10 in the great part of the flame. These $\mathrm{Ka}$ values fall within the limit of validity of the flamelet assumption made in the approach adopted to model the flame/turbulence interaction [19].

In order to investigate into the role of the sgs vortices, a simulation was run by assuming $\Xi_{\Delta}=1$ in Eq. (4), thus neglecting their effect on the flame propagation.

In Fig. 3, the time sequence of the reaction progress variable maps as obtained with $\Xi_{\Delta}=1$ (Fig. 3c) can be compared to the experimental images (Fig. 3a) and the previously computed maps (Fig. 3b). The flame arrival times are significantly different from the experimental times (the root mean square value of the difference between experimental and model results is around $11 \mathrm{~ms}$ ), given that the flame propagation with $\Xi_{\Delta}=1$ is obviously slower.

However, in this case, the shape and structure of the flame are very similar to the predictions obtained by the "full" sgs combustion model (i.e., by evaluating $\Xi_{\Delta}$ according to Charlette et al. [36]) (Fig. 3b): the flame front is only wrinkled at the wake of the first obstacle, while pockets are formed when the flame burns downstream of the second and third obstructions.

In Fig. 4 and Fig. 5, the flame speed profile and the pressure history as obtained with $\Xi_{\Delta}=1$ are also reported. Concerning the trend of the flame speed, it can be observed 
that it is the same as the "full" simulation: the acceleration-deceleration step around each obstacle is well simulated in both cases. The only difference is quantitative: the flame speed is lower when the role of the sgs vortices is neglected. According to the quantitative trend of the sgs wrinkling factor shown in Fig. 8a, this difference increases the further the flame progresses towards the second and third obstacles.

The pressure history as computed with $\Xi_{\Delta}=1$ reflects the corresponding trend of the flame speed, the pressure peak being significantly lower (the maximum overpressure is around $65 \%$ lower than in the experiment) and delayed (of around $15 \mathrm{~ms}$ ). Therefore, the increase of the combustion rate due to the contribution of the small scale vortices is also necessary for the correct prediction of the pressure peak.

These results show that the role of the unresolved turbulence is to enhance the flame speed and the pressure rise, but the main mechanisms driving the flame behaviour are still dominated by the large scale vortices that are directly solved.

This conclusion is obviously valid within the limits of the present investigation on a flame that remains a deflagration front. Extrapolation to conditions in which phenomena of flame instability start to dominate the scene, leading to transition into fast deflagration and/or detonation, is not straightforward.

\subsection{Regimes of Flame/Vortex Interaction}

From the above described results, it turns out that the main features of the unsteady flame propagation around the three repeated obstacles are mainly controlled by the interaction between the flame and the large scale vortices, which are measured by PIV and directly computed by LES. Therefore, important information may be obtained by focusing on such an interaction. 
Poinsot et al. [45] have proposed a diagram in which it is possible to correlate the vortex properties to the intensity of the flame/vortex interaction, and then to the combustion regime. This diagram has been developed from direct numerical simulations of interactions between a counter-rotating vortex pair and a stable, flat laminar flame front normal to the vortex displacement direction. The experimental studies by Renard et al. [46] and Samaniego and Mantel [47] have complemented the diagram that is shown in Fig. 9 (from Renard et al. [48]).

The vortex properties have been identified by means of two dimensionless ratios: the vortex core diameter to flame thickness ratio, $2 R / \delta_{f}$, and the maximum vortex rotational velocity to laminar burning velocity ratio, $u_{\theta}{ }^{\max } / S_{l}$.

In dependence on the vortex core diameter and rotational velocity, four distinct regimes have been observed which differ for the effects produced by the vortices on the flame surface and structure: no-effect regime (the flame is not affected by the vortex which is too small and/or too slow); wrinkled flame regime (the flame/vortex interaction produces wrinkling of the flame surface); pocket formation regime (the flame structure is disturbed by the vortex and isolated flame pockets are found); quenching regime (the vortex disrupts the flame by quenching it). Similar regimes of flame/vortex interaction have been found in the experiments by Roberts and co-workers $[49,50]$.

During the unsteady flame propagation around the obstacles, the vortices are induced by the progression of the flame itself (Fig. 6). Although their formation is somewhat controlled (a single main vortex sheds from each obstacle side, with well defined size and velocity), the subsequent interaction with the flame is more complex than that analysed by Poinsot et al. [45]. However, since no diagram has been set for quantifying 
the regimes of flame/vortex interaction in our configuration, an attempt is here made to use and test the diagram of Fig. 9 that is the "nearest" one.

To this end, we evaluated the diameter of the vortex core, $2 R$, and its rotational velocity, $u_{\theta}{ }^{\max }$, from both PIV and LES (with Charlette et al. [36]) data of the velocity vector fields ahead of the flame front at the wake of each obstruction.

The values obtained are reported in Table 1 . Both the experimental and numerical results confirm that the vortex dimension and velocity increase during the propagation through the three obstacles.

The $2 R / \delta_{f}$ and $u_{\theta \max } / S_{l}$ ratios were computed by assuming, for the stoichiometric methane/air flame used, a flame thickness, $\delta_{f}$, equal to $0.4 \mathrm{~mm}$ and a laminar burning velocity, $S_{l}$, equal to $0.41 \mathrm{~m} / \mathrm{s}$ [43,44] (Table 1). The flame thickness was determined through the one-dimensional CHEMKIN-based PREMIX code [51] in which the detailed GRI-Mech 3.0 reaction scheme [52] was implemented. Particularly, the flame thickness was obtained from the temperature profile. It was defined as the spatial distance between the unburned and burned conditions if the rate of temperature change corresponded to its maximum gradient throughout the transition [53].

The LES data related to the vortex size and velocity were also non-dimensionalised by using the parameters of the filtered flame and, more precisely, a flame thickness of the same order as the filter size $\Delta \approx 2 \mathrm{~mm}$, and a burning velocity equal to $\Xi_{\Delta} S_{l}$ (Table 1 ). The points have been placed on the diagram of Fig. 9 (squares: experiment; circles: LES; triangles: LES with the parameters of the filtered flame). There is agreement between experiment and simulations in that the vortices interact with the propagating flame in the wrinkled flame regime at the wake of the first obstacle, and in the pocket formation regime at the wake of the second and third obstacles. 
In Fig. 9, the points as calculated for the case $\Xi_{\Delta}=1$ are also shown (diamonds). It appears that, by neglecting the effects of the sgs turbulence on the flame propagation, the vortices are smaller and slower. However, the regimes of interaction between the flame and the large vortices at each obstacle wake are identified in close proximity to the experimental and previously modelled results.

These results are also consistent with the images of the propagating flame (Fig. 3), which show that only the flame surface, and not even the flame structure, is perturbed (wrinkled) by the vortex at the first obstacle. It is only from the second obstacle that the eddies become able to disrupt the continuity of the front, giving rise to the formation of flame pockets. This modification of the flame structure in turn enhances the flame propagation, as the flame burns through both the main front and the pockets themselves. It is worth emphasising that the transition from the wrinkled flame regime to the pocket formation regime is here driven by the resolved large scale vortices. Therefore, such a transition does not mean that the combustion model, which is based on the flamelet concept, overshoots its limits.

\section{Summary and Conclusions}

The unsteady premixed flame propagation around three repeated obstacles in a small scale vented combustion chamber has been studied by means of a combined use of advanced numerical and experimental techniques.

An LES model has been developed and coupled to the power-law flame wrinkling model by Charlette et al. [36] in the context of the flame surface density formalism. The model predictions have been compared to previous experimental data [1] that have been here extended to include simultaneous flame and PIV measurements of the flow field 
within the wake of each obstacle. A satisfactory agreement between numerical and experimental results has been obtained in terms of shape of the propagating flame, flame arrival times, spatial profile of the flame speed, pressure time history and velocity vector fields.

Once validated, the LES model has been used to study the role of the large scale vortices, in relation to that of the small sub-grid scale (sgs) vortices, on the features of the flame propagation. To achieve this, large eddy simulations have also been run with the effect of the sgs combustion model eliminated (i.e., by assuming the sub-grid wrinkling factor as constant and equal to 1 during the entire propagation).

The results obtained have demonstrated that the large scale vortices, which are measured by PIV and directly resolved by LES, play the dominant role in dictating all trends, including the evolution of the flame structure along the path. Conversely, the sgs vortices do not affect the qualitative trends. However, it is essential to model their effects on the combustion rate to achieve quantitative predictions for the flame speed and the pressure peak.

From both PIV and LES data, the regimes of flame/vortex interaction at the wake of each obstacle have been identified on the diagram by Poinsot et al. [45]. The experimental and numerical results have shown that the regimes present within the interaction range from the wrinkled flame regime (at the wake of the first obstacle) up to the pocket formation regime (at the wake of the second and third obstacles). The identification of these regimes is consistent with the observation of the flame behaviour. 


\section{Acknowledgements}

Valeria Di Sarli gratefully acknowledges the kind hospitality from Wolfson School of Mechanical and Manufacturing Engineering (Loughborough University, UK) where the experimental work described in the paper was performed. Partial support for her stay in Loughborough during the $\mathrm{PhD}$ activity was provided by Consiglio Nazionale delle Ricerche (CNR, Italy) through the Short-Term Mobility Program 2007.

The authors wish to thank the reviewers for their thorough and careful examination of the manuscript draft and for several suggestions now incorporated in the paper. 


\section{Nomenclature}

$c$, reaction progress variable

$D$, diffusion coefficient

$K a$, Karlovitz number

$M$, parameter to measure the turbulence resolution

$R_{i g n}$, radius of the ignition patch

$2 R$, vortex diameter

$R e_{\Delta}$, sub-grid scale turbulent Reynolds number

$S_{l}$, laminar burning velocity

$u$, velocity

$u_{\Delta}^{\prime}$, sub-grid scale turbulent velocity

$u_{\Delta_{-} i g n}^{\prime}$, sub-grid scale turbulent velocity of the ignition patch

$u_{\theta}^{\max }$, vortex rotational velocity

$w$, local displacement speed

$y^{+}$, y plus at the first grid point

\section{Greek symbols}

$\beta$, exponent of the sub-grid scale combustion model

$\Gamma$, efficiency function

$\Delta$, filter size

$\delta_{f}$, laminar flame thickness

$\eta_{c}$, inner cut-off flame scale

$\Xi_{\Delta}$, sub-grid scale flame wrinkling factor

$\rho$, fluid density 
$\rho_{0}$, density of the unburned gas

$\Sigma$, sub-grid scale flame surface density

$\dot{\omega}_{c}$, reaction rate 


\section{References}

1. Patel, S.N.D.H., Jarvis, S., Ibrahim, S.S., Hargrave, G.K.: An experimental and numerical investigation of premixed flame deflagration in a semiconfined explosion chamber. Proc. Combust. Inst. 29, 1849-1854 (2002)

2. Lindstedt, R.P., Sakthitharan, V.: Time resolved velocity and turbulence measurements in turbulent gaseous explosions. Combust. Flame 114, 469-483 (1998)

3. Fairweather, M., Hargrave, G.K., Ibrahim, S.S., Walker, D.G.: Studies of premixed flame propagation in explosion tubes. Combust. Flame 116, 504-518 (1999)

4. Masri, A.R., Ibrahim, S.S., Nehzat, N., Green, A.R.: Experimental study of premixed flame propagation over various solid obstructions. Exp. Therm. Fluid Sci. 21, 109-116 (2000)

5. Masri, A.R., Ibrahim, S.S., Cadwallader, B.J.: Measurements and large eddy simulation of propagating premixed flames. Exp. Therm. Fluid Sci. 30, 687-702 (2006)

6. Ibrahim, S.S., Hargrave, G.K., Williams, T.C.: Experimental investigation of flame/solid interactions in turbulent premixed combustion. Exp. Therm. Fluid Sci. 24, 99-106 (2001)

7. Hargrave, G.K., Jarvis, S., Williams, T.C.: A study of transient flow turbulence generation during flame/wall interactions in explosions. Meas. Sci. Technol. 13, 1036-1042 (2002) 
8. Kirkpatrick, M.P., Armfield, S.W., Masri, A.R., Ibrahim, S.S.: Large eddy simulation of a propagating turbulent premixed flame. Flow Turbul. Combust. 70, 1$19(2003)$

9. Jarvis, S., Hargrave, G.K.: A temporal PIV study of flame/obstacle generated vortex interactions within a semi-confined combustion chamber. Meas. Sci. Technol. 17, $91-100(2006)$

10. Long, E.J., Hargrave, G.K., Jarvis, S., Justham, T., Halliwell, N.: Characterisation of the interaction between toroidal vortex structures and flame front propagation. J. Phys. Conf. Ser. 45, 104-111 (2006)

11. Park, D.J., Green, A.R., Lee, Y.S., Chen, Y.-C.: Experimental studies on interactions between a freely propagating flame and single obstacles in a rectangular confinement. Combust. Flame 150, 27-39 (2007)

12. Wolfrum, J.: Lasers in combustion: From basic theory to practical devices. Proc. Combust. Inst. 27, 1-41 (1998)

13. Hassel, E.P., Linow, S.: Laser diagnostics for studies of turbulent combustion. Meas. Sci. Technol. 11, R37-R57 (2000)

14. Kohse-Höinghaus, K., Barlow, R.S., Aldén, M., Wolfrum, J.: Combustion at the focus: Laser diagnostics and control. Proc. Combust. Inst. 30, 89-123 (2005)

15. Barlow, R.S.: Laser diagnostics and their interplay with computations to understand turbulent combustion. Proc. Combust. Inst. 31, 49-75 (2007)

16. Hasegawa, T., Michikami, S., Nomura, T., Gotoh, D., Sato, T.: Flame development along a straight vortex. Combust. Flame 129, 294-304 (2002) 
17. Filatyev, S.A., Thariyan, M.P., Lucht, R.P., Gore, J.P.: Simultaneous stereo particle image velocimetry and double-pulsed planar laser-induced fluorescence of turbulent premixed flames. Combust. Flame 150, 201-209 (2007)

18. Janicka, J., Sadiki, A.: Large eddy simulation of turbulent combustion systems. Proc. Combust. Inst. 30, 537-547 (2005)

19. Poinsot, T., Veynante, D.: Theoretical and Numerical Combustion. Second edition, R.T. Edwards, Inc., Philadelphia, PA, USA (2005)

20. Pitsch, H.: Large-eddy simulation of turbulent combustion. Annu. Rev. Fluid Mech. 38, 453-482 (2006)

21. Ji, J., Gore, J.P.: Flow structure in lean premixed swirling combustion. Proc. Combust. Inst. 29, 861-867 (2002)

22. Archer, S., Gupta, A.K.: Effect of swirl on flow dynamics in unconfined and confined gaseous fuel flames. AIAA Paper, AIAA-2004-813 (2004)

23. Selle, L., Lartigue, G., Poinsot, T., Koch, R., Schildmacher, K.-U., Krebs, W., Prade, B., Kaufmann, P., Veynante, D.: Compressible large eddy simulation of turbulent combustion in complex geometry on unstructured meshes. Combust. Flame 137, 489-505 (2004)

24. Roux, S., Lartigue, G., Poinsot, T., Meier, U., Bérat, C.: Studies of mean and unsteady flow in a swirled combustor using experiments, acoustic analysis, and large eddy simulations. Combust. Flame 141, 40-54 (2005)

25. Wicksall, D.M., Agrawal, A.K., Schefer, R.W., Keller, J.O.: The interaction of flame and flow field in a lean premixed swirl-stabilized combustor operated on $\mathrm{H}_{2} / \mathrm{CH}_{4}$ /air. Proc. Combust. Inst. 30, 2875-2883 (2005) 
26. Boudier, G., Gicquel, L.Y.M., Poinsot, T., Bissières, D., Bérat, C.: Comparison of LES, RANS and experiments in an aeronautical gas turbine combustion chamber. Proc. Combust. Inst. 31, 3075-3082 (2007)

27. Richard, S., Colin, O., Vermorel, O., Benkenida, A., Angelberger, C., Veynante, D.: Towards large eddy simulation of combustion in spark ignition engines. Proc. Combust. Inst. 31, 3059-3066 (2007)

28. Boger, M., Veynante, D., Boughanem, H., Trouvé, A.: Direct numerical simulation analysis of flame surface density concept for large eddy simulation of turbulent premixed combustion. Proc. Combust. Inst. 27, 917-925 (1998)

29. Bray, K.N.C.: The challenge of turbulent combustion. Proc. Combust. Inst. 26, 1-26 (1996)

30. Libby, P.A., Williams, F.A. (eds.): Turbulent Reacting Flows. Academic Press, New York (1993)

31. Pope, S.B.: Turbulent flows. Cambridge University Press (2000)

32. Lilly, D.K.: A proposed modification of the Germano subgrid-scale closure method. Phys. Fluids A 4, 633-635 (1992)

33. Kim, S.-E.: Large eddy simulation using unstructured meshes and dynamic subgridscale turbulence models. AIAA Paper, AIAA-2004-2548 (2004)

34. Loitsyanskiy, L.G.: Mechanics of Liquids and Gases. $6^{\text {th }}$ edition, Begell House, New York (1995)

35. Trouvé, A., Poinsot, T.: The evolution equation for the flame surface density. J. Fluid Mech. 278, 1-31 (1994) 
36. Charlette, F., Meneveau, C., Veynante, D.: A power-law flame wrinkling model for LES of premixed turbulent combustion. Part I: Non-dynamic formulation and initial tests. Combust. Flame 131, 159-180 (2002)

37. Colin, F., Veynante, D., Poinsot, T.: A thickened flame model for large eddy simulations of turbulent premixed combustion. Phys. Fluids 12, 1843-1863 (2000)

38. Abdel-Gayed, R.G., Bradley, D.: A two-eddy theory of premixed turbulent flame propagation. Philos. Trans. R. Soc. Lond. Ser. A 301, 1-25 (1981)

39. Fluent 6.3.26, 2007, Fluent Inc., Lebanon, NH (USA), website: www.fluent.com (accessed on $26^{\text {th }}$ March 2008)

40. Pope, S.B.: Ten questions concerning the large-eddy simulation of turbulent flows. New J. Phys. 6, 35 (2004)

41. Kader, B.: Temperature and concentration profiles in fully turbulent boundary layers. Int. J. Heat Mass Transfer 24, 1541-1544 (1981)

42. Boudier, P., Henriot, S., Poinsot, T., Baritaud, T.: A model for turbulent flame ignition and propagation in piston engines. Proc. Combust. Inst. 24, 503-510 (1992)

43. Yu, G., Law, C.K., Wu, C.K.: Laminar flame speeds of hydrocarbon + air mixtures with hydrogen addition. Combust. Flame 63, 339-347 (1986)

44. Di Sarli, V., Di Benedetto, A.: Laminar burning velocity of hydrogen-methane/air premixed flames. Int. J. Hydrogen Energy 32, 637-646 (2007)

45. Poinsot, T., Veynante, D., Candel, S.: Quenching processes and premixed turbulent combustion diagrams. J. Fluid Mech. 228, 561-606 (1991)

46. Renard, P.-H., Rolon, J.C., Thévenin, D., Candel, S.: Wrinkling, pocket formation and double premixed flame interaction processes. Proc. Combust. Inst. 27, 659-666 (1998) 
47. Samaniego, J.-M., Mantel, T.: Fundamental mechanisms in premixed turbulent flame propagation via flame-vortex interactions. Part I: Experiment. Combust. Flame 118, 537-556 (1999)

48. Renard, P.-H., Thévenin, D., Rolon, J.C., Candel, S.: Dynamics of flame/vortex interactions. Prog. Energy Combust. Sci. 26, 225-282 (2000)

49. Roberts, W.L., Driscoll, J.F.: A laminar vortex interacting with a premixed flame: Formation of pockets of reactants. Combust. Flame 87, 245-256 (1991)

50. Roberts, W.L., Driscoll, J.F., Drake, M.C., Goss, L.P.: Images of the quenching of a flame by a vortex - to quantify regimes of turbulent combustion. Combust. Flame 94, 58-69 (1993)

51. Kee, R.J., Grcar, J.F., Smooke, M.D., Miller, J.A.: A Fortran program for modeling steady laminar one-dimensional premixed flames. Sandia Report SAND85-8240 (1985)

52. Bowman, C.T., Frenklach, M., Gardiner, W.C., Smith, G.P.: The “GRI-Mech 3.0” chemical kinetic mechanism. http://www.me.berkeley.edu/gri_mech (1999) (accessed on $30^{\text {th }}$ June 2008)

53. Sun, C.J., Sung, C.J., He, L., Law, C.K.: Dynamics of weakly stretched flames: Quantitative description and extraction of global flame parameters. Combust. Flame 118, 108-128 (1999) 
Table 1 - Experimental results and LES predictions at the wake of each obstacle: vortex core diameter, $2 \mathrm{R}$, maximum rotational velocity, $\mathbf{u}_{\theta}{ }^{\mathrm{max}}, 2 \mathrm{R} / \delta_{\mathrm{f}}$ and $\mathrm{u}_{\theta}{ }^{\mathrm{max}} / \mathrm{S}_{\mathrm{I}}$ ratios; the LES data are also non-dimensionalised with the parameters of the filtered flame, i.e., $\Delta$ and $\Xi_{\Delta} S_{I}$

\begin{tabular}{|c|c|c|c|c|c|c|c|c|c|c|c|c|}
\hline & \multicolumn{4}{|c|}{ Experiment } & \multicolumn{8}{|c|}{ LES } \\
\hline Obstacle & $\begin{array}{c}\mathbf{2 R} \\
{[\mathrm{mm}]}\end{array}$ & $\begin{array}{l}\mathbf{u}_{\theta}^{\max } \\
{[\mathrm{m} / \mathrm{s}]}\end{array}$ & $2 \mathrm{R} / \delta_{\mathrm{f}}$ & $\mathbf{u}_{\theta}^{\max } / S_{\mathbf{l}}$ & $\begin{array}{c}2 \mathbf{R} \\
{[\mathrm{mm}]}\end{array}$ & $\begin{array}{l}\mathbf{u}_{\theta}{ }^{\max } \\
{[\mathrm{m} / \mathrm{s}]}\end{array}$ & $2 \mathrm{R} / \delta_{\mathrm{f}}$ & $\mathbf{u}_{\theta}{ }^{\max } / \mathrm{S}_{\mathbf{I}}$ & $\begin{array}{c}\Delta \\
{[\mathrm{mm}]}\end{array}$ & $\Xi_{\Delta}$ & $2 \mathrm{R} / \Delta$ & $\mathbf{u}_{\theta}{ }^{\max } /\left(\Xi_{\Delta} S_{\mathrm{l}}\right)$ \\
\hline First & 7 & 3.5 & 17.5 & 8.5 & 6.5 & 3 & 16.25 & 7.3 & 2 & 1.7 & 3.25 & 4.3 \\
\hline Second & 15 & 12 & 37.5 & 29.3 & 16 & 14 & 40 & 34 & 2 & 2.2 & 8 & 15.5 \\
\hline Third & 30 & 23 & 75 & 56 & 32 & 26 & 80 & 63.4 & 2 & 3 & 16 & 21 \\
\hline
\end{tabular}




\section{Figure Captions}

Figure 1 - Schematic diagram of the explosion chamber with PIV system.

Figure 2- Time evolution of the flame location at varying the ignition patch characteristics.

Figure 3 - Time sequence of the flame images at the central plane of the combustion chamber: HSLSFV results by Patel et al. [1] (a), LES calculations of the reaction progress variable maps as obtained with the combustion sub-model by Charlette et al. [36] (b) and by assuming $\Xi_{\Delta}=1$ (c).

Figure 4 - Measured flame speed profile along the axial distance from the ignition face and corresponding LES predictions as calculated with the combustion sub-model by Charlette et al. [36] and by assuming $\Xi_{\Delta}=1$ (the black rectangles along the x-axis indicate the positions of the three obstacles).

Figure 5 - Measured pressure time history at the bottom end of the combustion chamber and corresponding LES predictions as calculated with the combustion sub-model by Charlette et al. [36] and by assuming $\Xi_{\Delta}=1$.

Figure 6 - Measured (bottom) and calculated (top) instantaneous velocity vector maps ahead of the front (vectors colored by velocity magnitude, $\mathrm{m} / \mathrm{s}$ ) at the wake of the first (a), second (b) and third (c) obstacles. The images were taken at the central plane of the combustion chamber.

Figure 7 - LES calculations of the vorticity magnitude (1/s) maps on the iso-surface $c=0.1$ at different propagation stages.

Figure 8 - Field profiles of the sub-grid scale wrinkling factor $\left(\Xi_{\Delta}\right.$, Eq. 5) (a) and the Karlovitz number (b), both conditioned on the reaction rate. The images were taken at the central plane of the combustion chamber. 
Figure 9 - Diagram of flame/vortex interactions by Poinsot et al. [45] including the current results at each obstacle wake (squares: experiment; circles: LES; triangles: LES non-dimensionalised with the parameters of the filtered flame; diamonds: LES with $\left.\Xi_{\Delta}=1\right)$. White, grey and black symbols correspond to the first, second and third obstacles, respectively. 


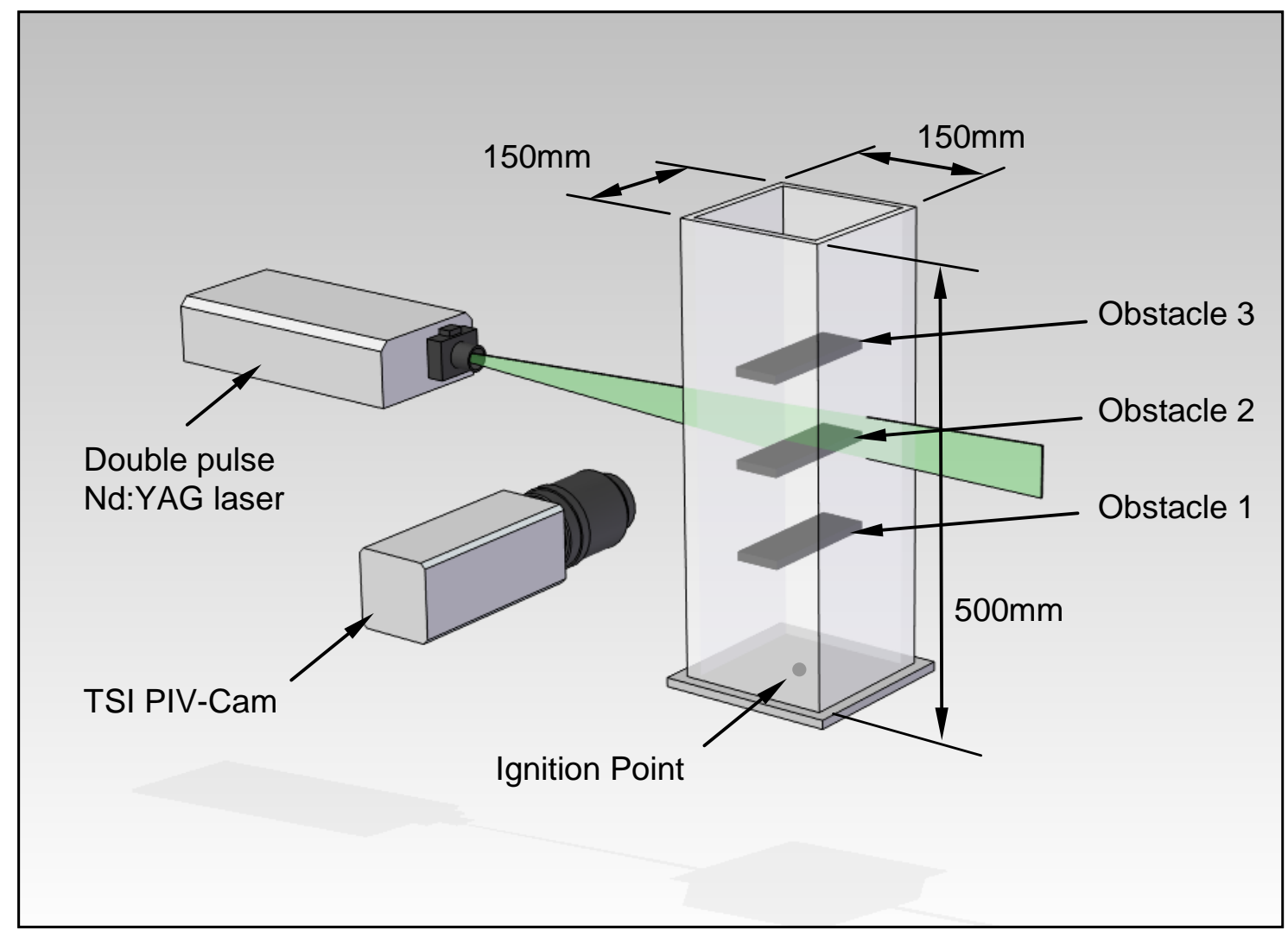

Figure 1 - Schematic diagram of the explosion chamber with PIV system. 


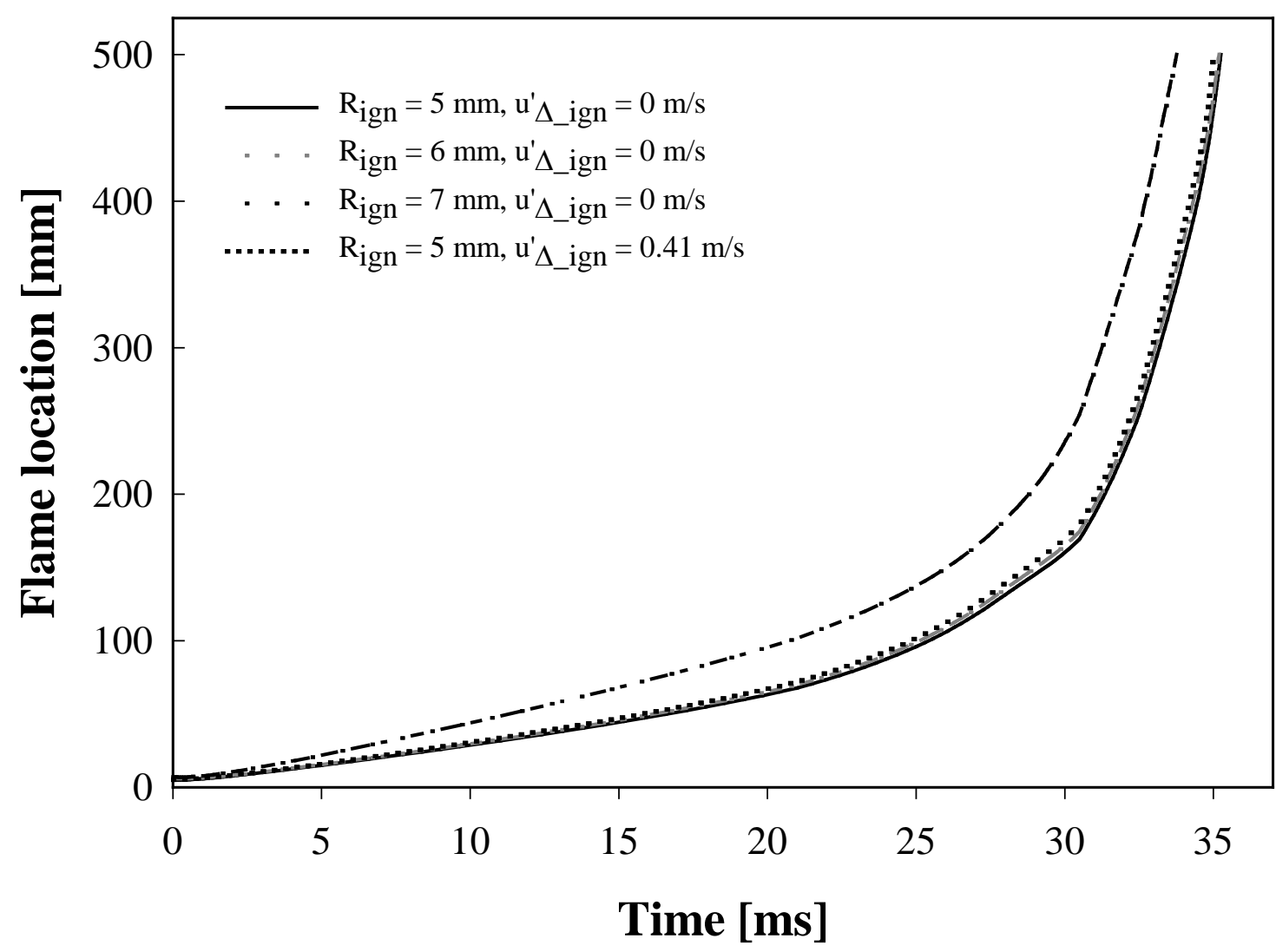

Figure 2 - Time evolution of the flame location at varying the ignition patch characteristics. 


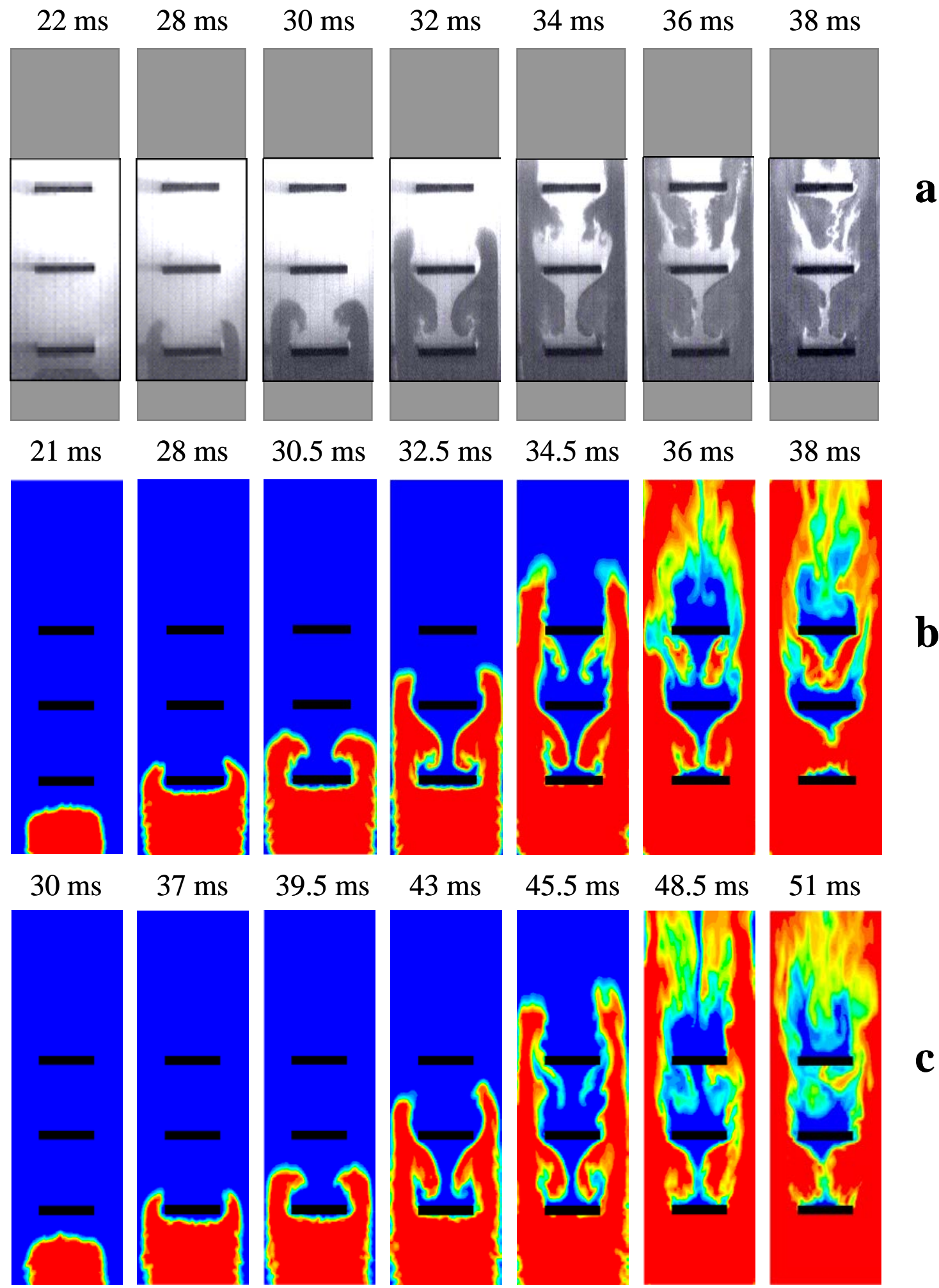

Figure 3 - Time sequence of the flame images at the central plane of the combustion chamber: HSLSFV results by Patel et al. [1] (a), LES calculations of the reaction progress variable maps as obtained with the combustion sub-model by Charlette et al. [36] (b) and by assuming $\Xi_{\Delta}=1$ (c). 


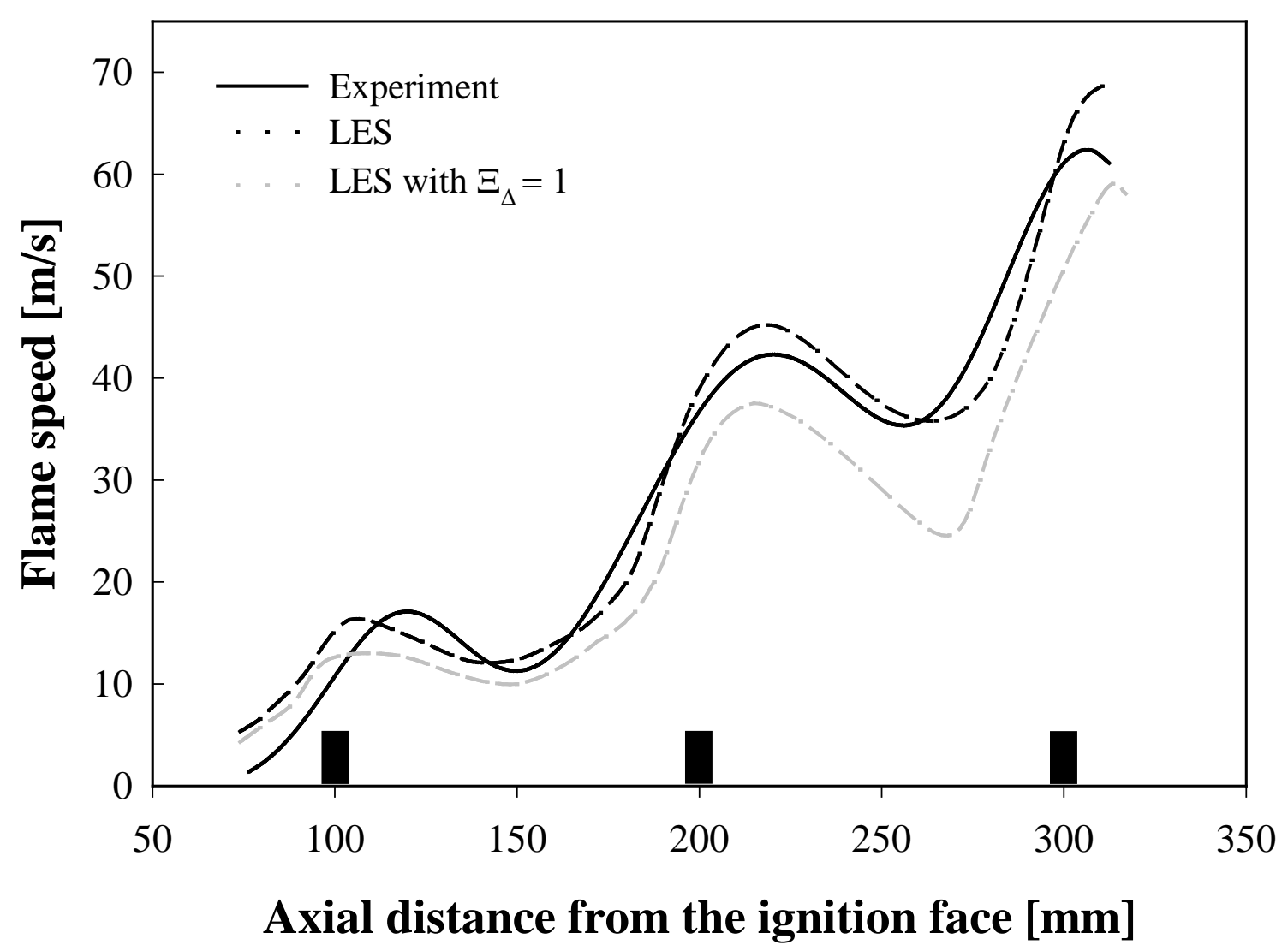

Figure 4 - Measured flame speed profile along the axial distance from the ignition face and corresponding LES predictions as calculated with the combustion sub-model by Charlette et al. [36] and by assuming $\Xi_{\Delta}=1$ (the black rectangles along the $x$-axis indicate the positions of the three obstacles). 


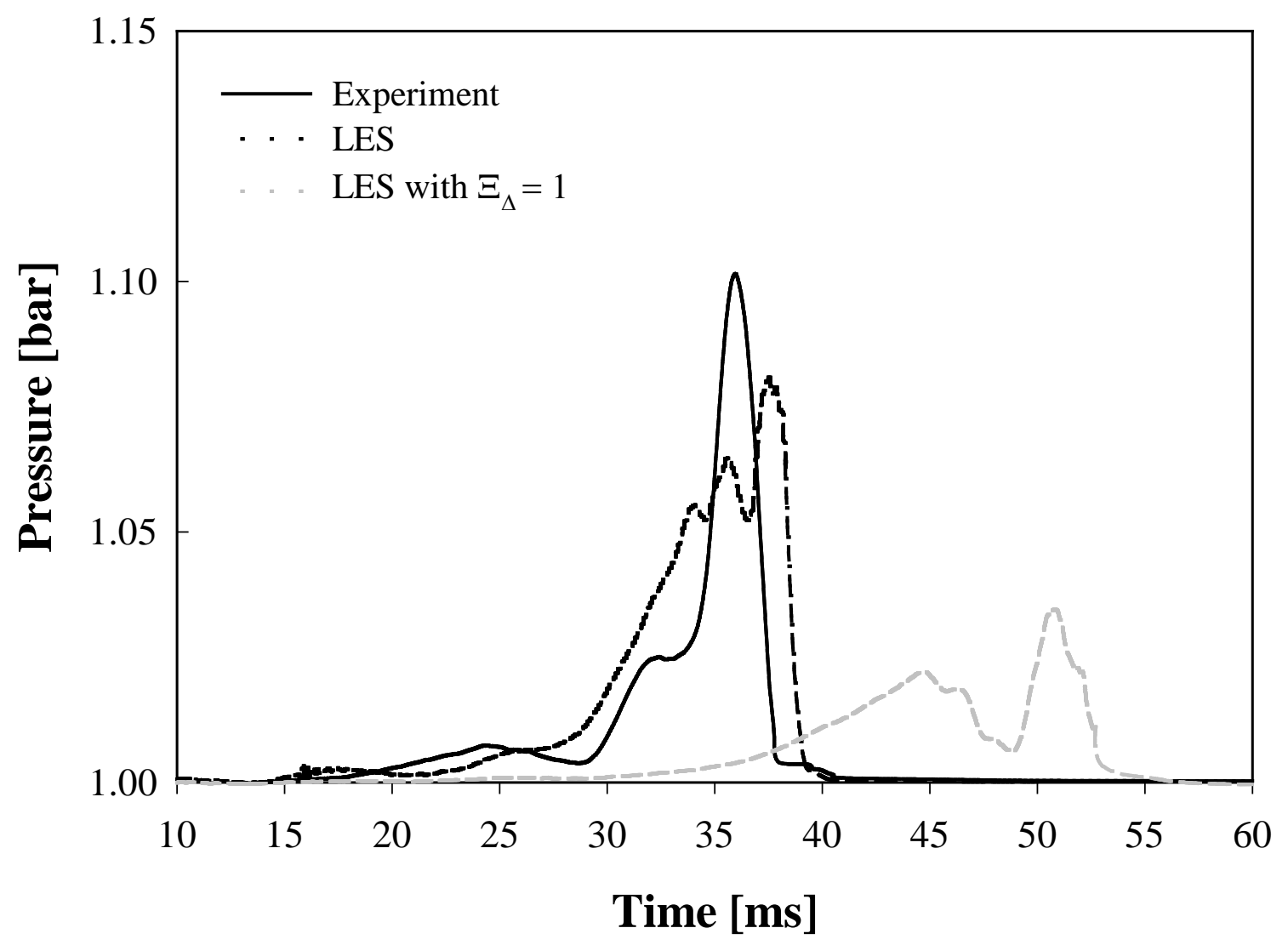

Figure 5 - Measured pressure time history at the bottom end of the combustion chamber and corresponding LES predictions as calculated with the combustion sub-model by Charlette et al. [36] and by assuming $\Xi_{\Delta}=1$. 

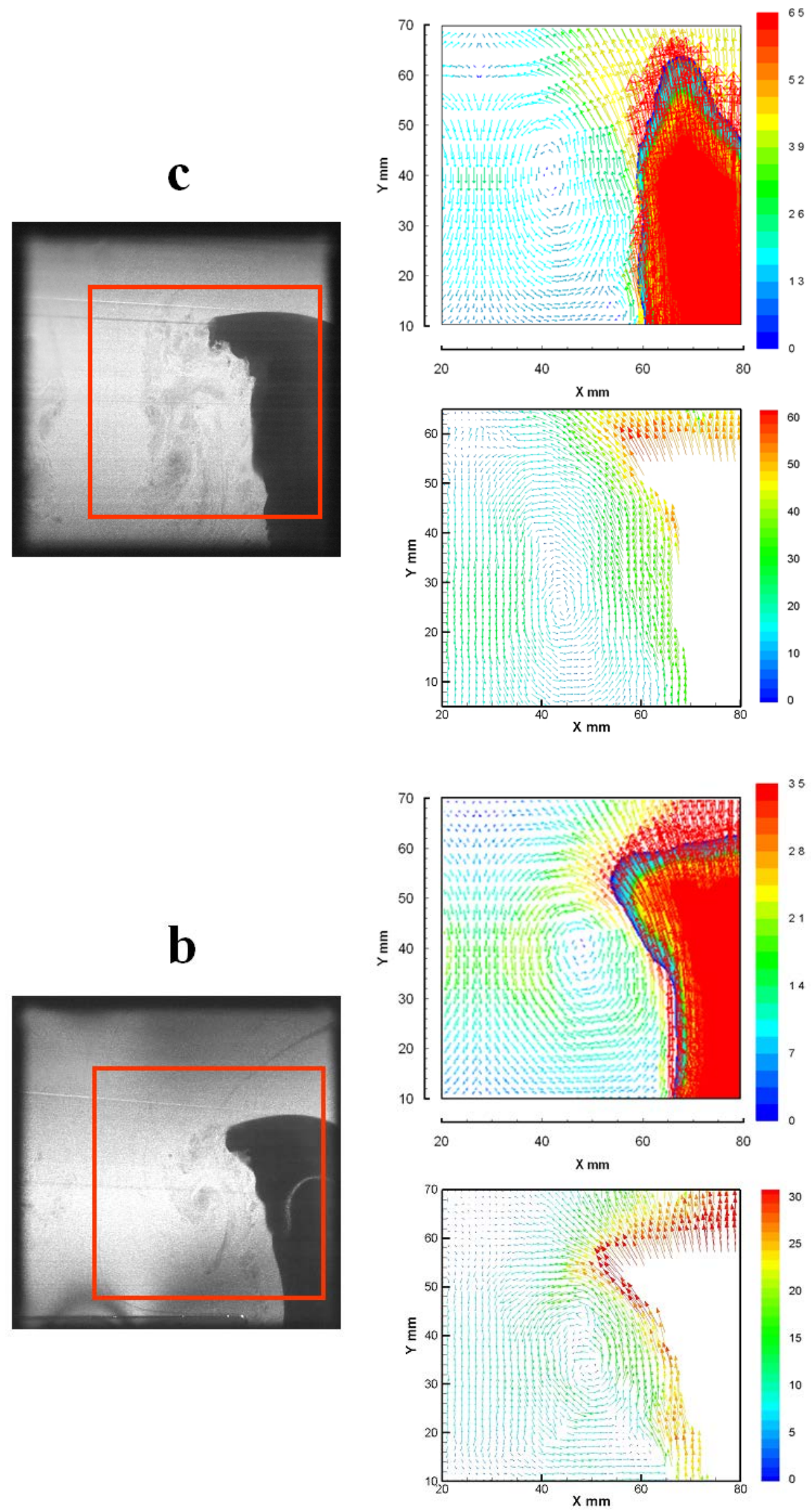

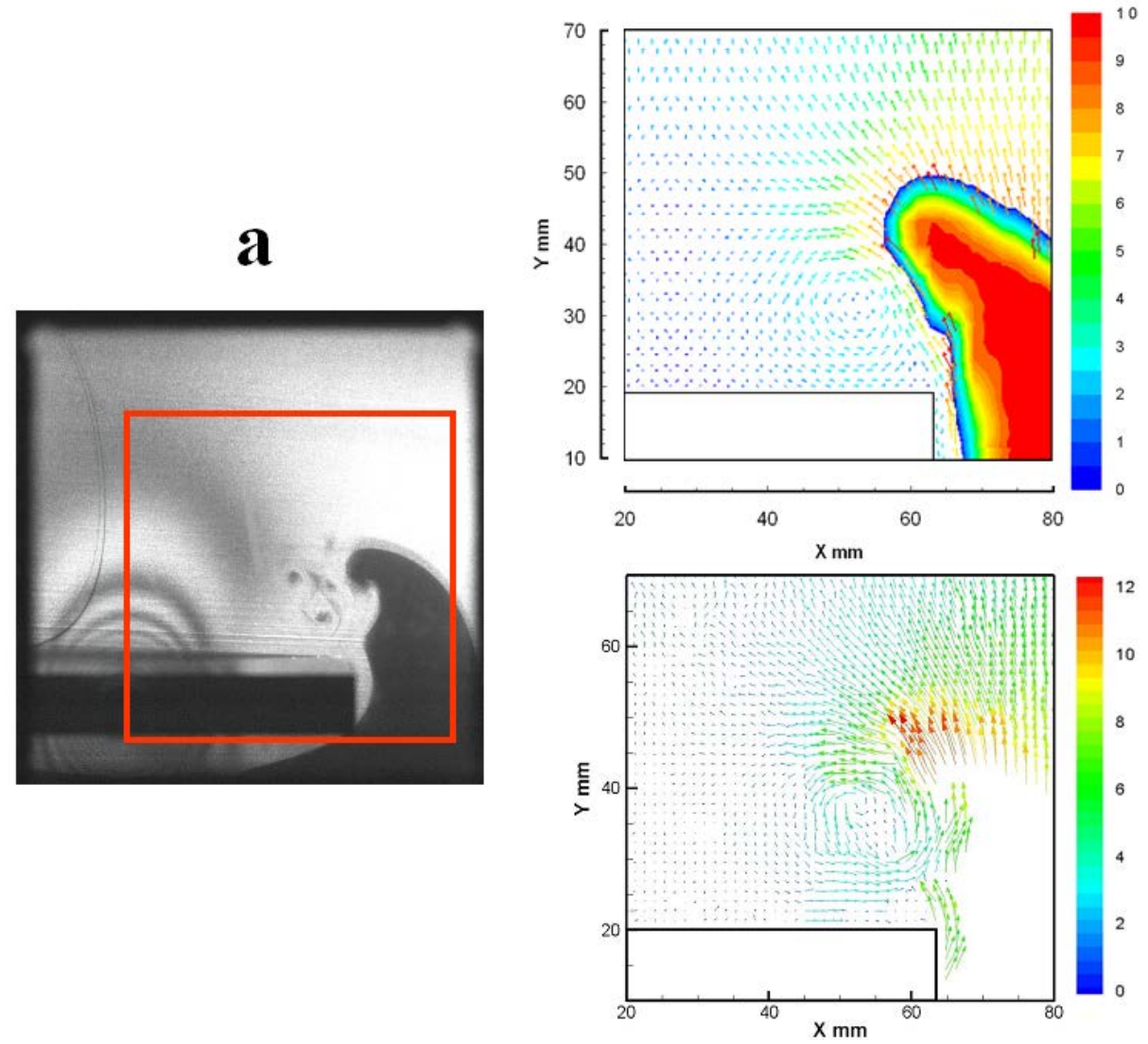

Figure 6 - Measured (bottom) and calculated (top) instantaneous velocity vector maps ahead of the front (vectors colored by velocity magnitude, $\mathrm{m} / \mathrm{s}$ ) at the wake of the first (a), second (b) and third (c) obstacles. The images were taken at the central plane of the combustion chamber. 
Isosurface $c=0.1$ colored

by vorticity magnitude $[1 / \mathrm{s}]$
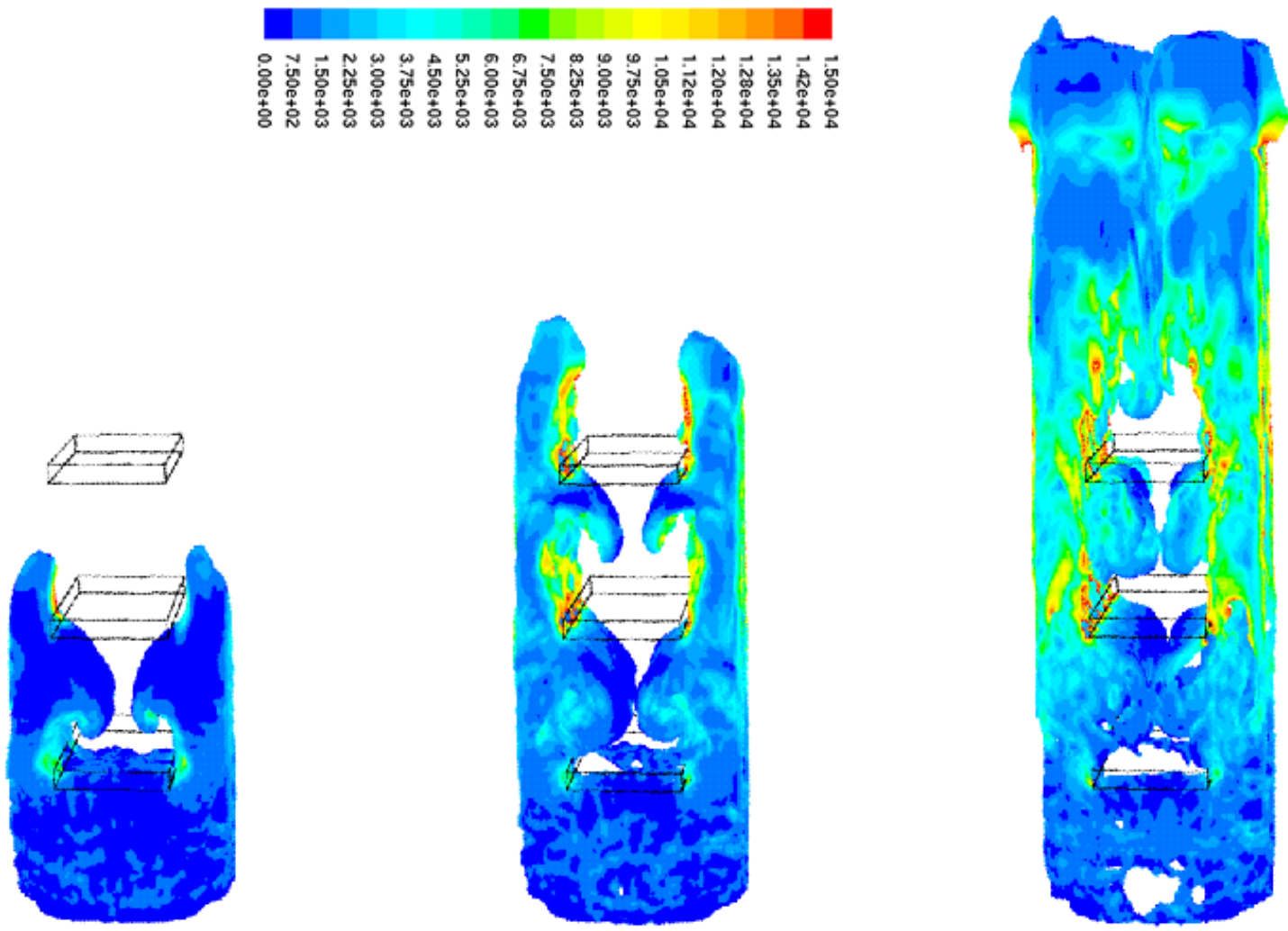

Figure 7 - LES calculations of the vorticity magnitude $(1 / \mathrm{s})$ maps on the iso-surface $c=0.1$ at different propagation stages. 


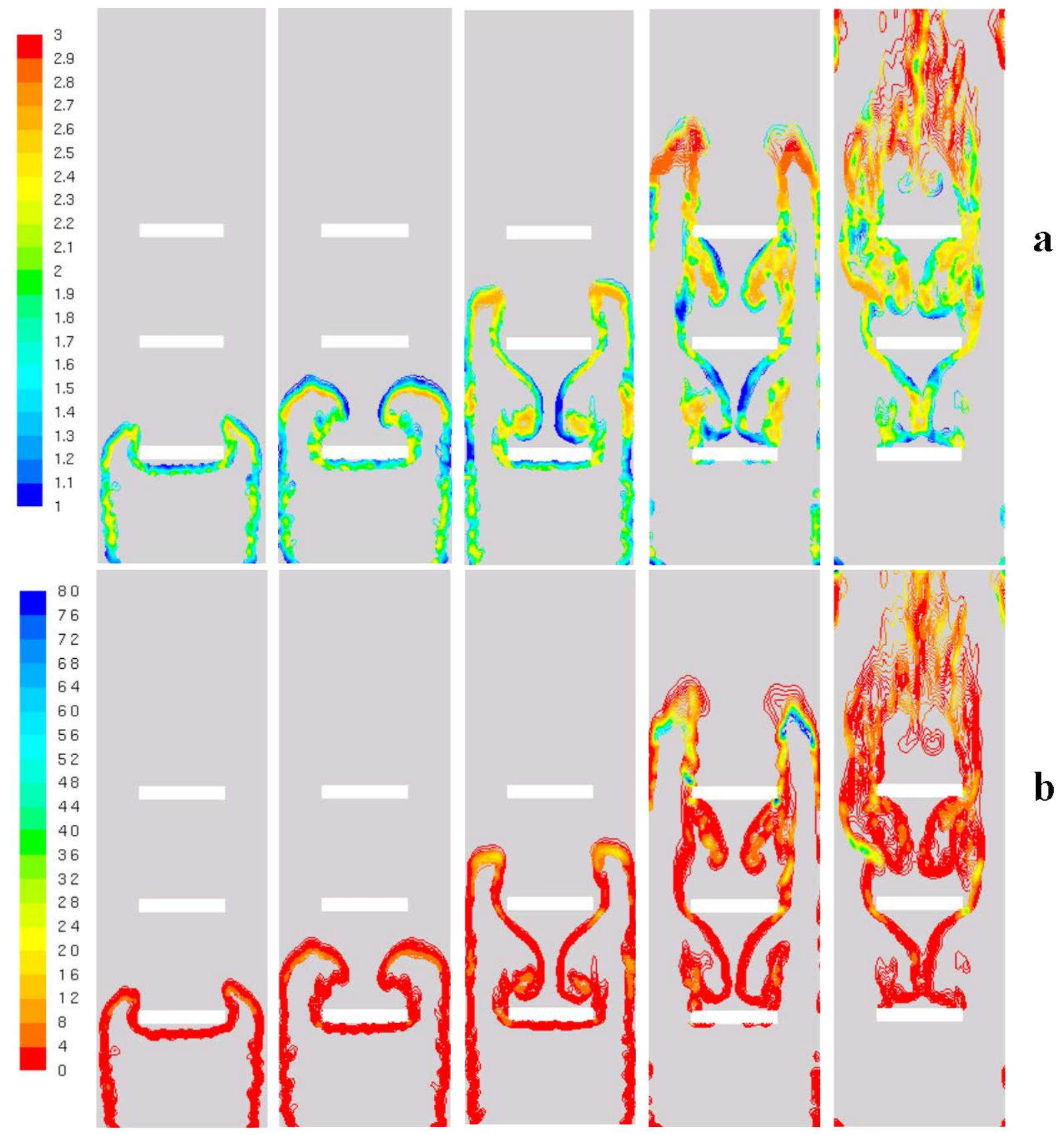

Figure 8 - Field profiles of the sub-grid scale wrinkling factor $\left(\Xi_{\Delta}\right.$, Eq. 5) (a) and the Karlovitz number (b), both conditioned on the reaction rate. The images were taken at the central plane of the combustion chamber. 


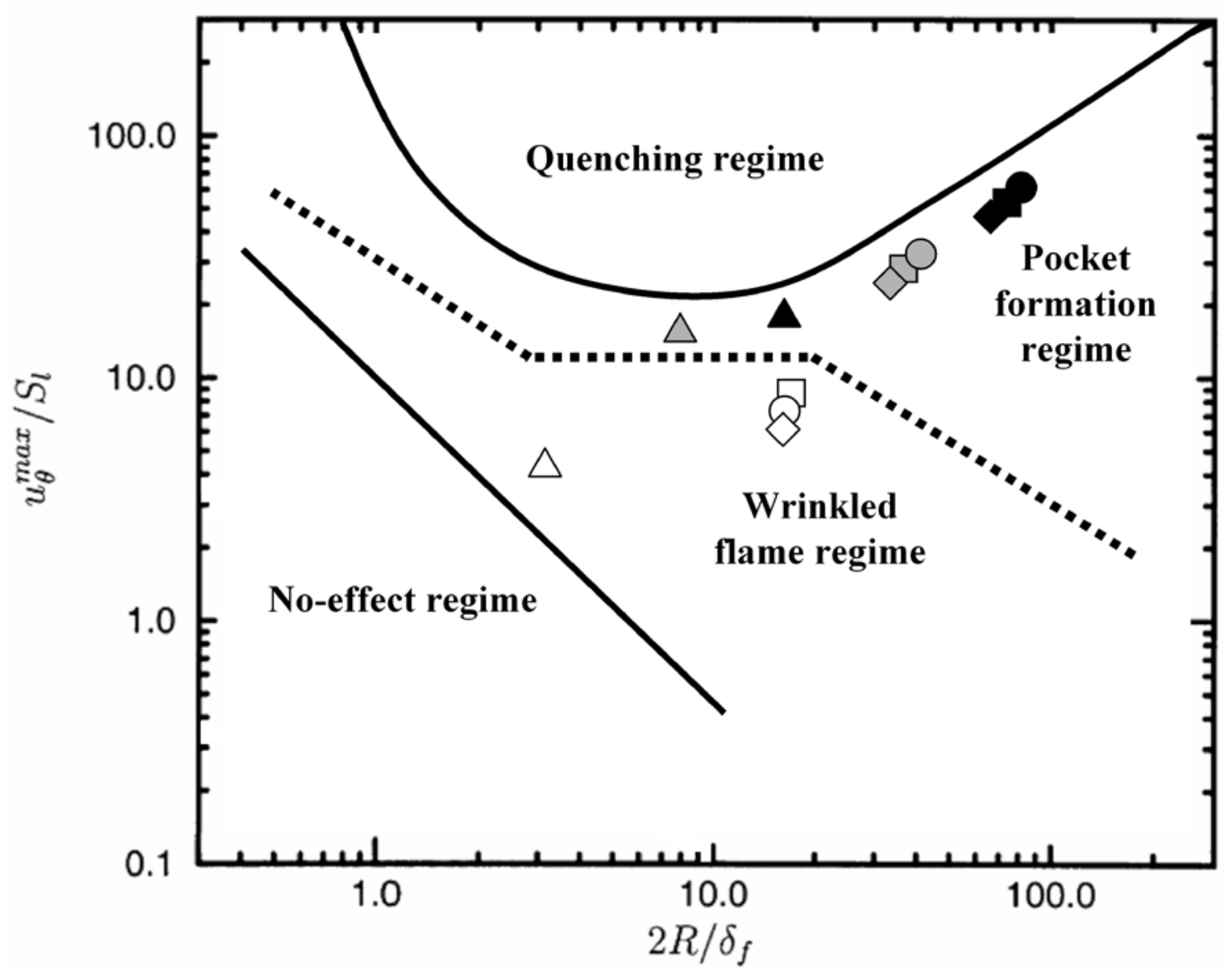

Figure 9 - Diagram of flame/vortex interactions by Poinsot et al. [45] including the current results at each obstacle wake (squares: experiment; circles: LES; triangles: LES nondimensionalised with the parameters of the filtered flame; diamonds: LES with $\Xi_{\Delta}=1$ ). White, grey and black symbols correspond to the first, second and third obstacles, respectively. 